 \\ TATRA \\ MOUNTaiNS \\ Mathematical Publications
}

\section{DENSITY TOPOLOGIES ON THE PLANE BETWEEN ORDINARY AND STRONG}

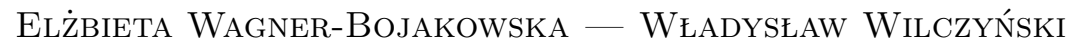

\begin{abstract}
Let $C_{0}$ denote the set of all non-decreasing continuous functions $f:(0,1] \rightarrow(0,1]$ such that $\lim _{x \rightarrow 0^{+}} f(x)=0$ and $f(x) \leq x$ for $x \in(0,1]$ and let $A$ be a measurable subset of the plane. We define the notion of a density point of $A$ with respect to $f$. This is a starting point to introduce the mapping $D_{f}$ defined on the family of all measurable subsets of the plane, which is so-called lower density. The mapping $D_{f}$ leads to the topology $\mathcal{T}_{f}$, analogously as for the density topology. The properties of the topologies $\mathcal{T}_{f}$ are considered.
\end{abstract}

Let $C_{0}$ denote the set of all non-decreasing continuous functions

$f:(0,1] \rightarrow(0,1]$ such that $\lim _{x \rightarrow 0^{+}} f(x)=0$ and $f(x) \leq x$ for $x \in(0,1]$.

Let $f \in C_{0}$ and let $A \subset \mathbb{R}^{2}$ be a measurable subset of the plane.

Definition 1. We will say that $(0,0)$ is a density point of $A$ with respect to a function $f$ if for each $\epsilon>0$ there exists $\delta>0$ such that for each $h, k \in(0, \delta)$ if $f(h) \leq k \leq h$ or $f(k) \leq h \leq k$ then

$$
\frac{m_{2}(A \cap([-h, h] \times[-k, k]))}{4 h k}>1-\epsilon .
$$

Obviously, the condition (11) is equivalent to the following inequality

$$
\frac{m_{2}\left(A^{\prime} \cap([-h, h] \times[-k, k])\right)}{4 h k}<\epsilon,
$$

where $A^{\prime}=\mathbb{R}^{2} \backslash A$.

We say that $\left(x_{0}, y_{0}\right)$ is a density point of $A$ with respect to a function $f$ if $(0,0)$ is a density point of $A-\left(x_{0}, y_{0}\right)=\left\{\left(x-x_{0}, y-y_{0}\right):(x, y) \in A\right\}$ with respect to $f$.

Obviously, if $f(x)=x$ for $x \in(0,1]$, then the notion of a density point with respect to $f$ coincides with the notion of the ordinary density point of a measurable subset of the plane.

2000 Mathematics Subject Classification: Primary 28A05; Secondary 54A05, 54A10. Keywords: density point, density topology, density point with respect to $f$. 


\section{ELŻBIETA WAGNER-BOJAKOWSKA — WŁADYSŁAW WILCZYŃSKI}

Theorem 2. Let $f \in C_{0}$ and let $A$ be a measurable subset of the plane. The point $(0,0)$ is a density point of $A$ with respect to $f$ if and only if for each $\epsilon>0$ there exists $n_{0} \in \mathbb{N}$ such that for all positive integers $n, m$ greater than $n_{0}$ if

$$
f\left(\frac{1}{n}\right) \leq \frac{1}{m} \leq \frac{1}{n} \quad \text { or } \quad f\left(\frac{1}{m}\right) \leq \frac{1}{n} \leq \frac{1}{m},
$$

then

$$
\frac{m_{2}\left(A \cap\left(\left[-\frac{1}{n}, \frac{1}{n}\right] \times\left[-\frac{1}{m}, \frac{1}{m}\right]\right)\right)}{\frac{4}{n m}}>1-\epsilon .
$$

Proof. " $\Longrightarrow$ " Let $\epsilon$ be an arbitrary positive number. From our assumption, there exists $\delta>0$ such that for each $h, k \in(0, \delta)$ if $f(h) \leq k \leq h$ or $f(k) \leq h \leq k$ then

$$
\frac{m_{2}(A \cap([-h, h] \times[-k, k]))}{4 h k}>1-\epsilon .
$$

Put $n_{0}=\left[\frac{1}{\delta}\right]+1$. Then $n_{0}-1 \leq 1 / \delta<n_{0}$, so $1 / n_{0}<\delta \leq 1 /\left(n_{0}-1\right)$. Let $n, m$ be arbitrary positive integers greater than $n_{0}$ and suppose that

$$
f\left(\frac{1}{n}\right) \leq \frac{1}{m} \leq \frac{1}{n} .
$$

Then put $h=\frac{1}{n}, k=\frac{1}{m}$. Obviously, $h, k \in(0, \delta)$ and $f(h) \leq k \leq h$; consequently, the inequality (11) holds which means that the condition (3) is fulfilled. If

the proof is analogous.

$$
f\left(\frac{1}{m}\right) \leq \frac{1}{n} \leq \frac{1}{m},
$$

" $"$ Let $\epsilon>0$. From our assumption, there exists $n_{0} \in \mathbb{N}, n_{0} \geq 4 / \epsilon$ such that for $n, m \in \mathbb{N}, n>n_{0}, m>n_{0}$, if

$$
f\left(\frac{1}{n}\right) \leq \frac{1}{m} \leq \frac{1}{n} \quad \text { or } \quad f\left(\frac{1}{m}\right) \leq \frac{1}{n} \leq \frac{1}{m},
$$

then

$$
\frac{m_{2}\left(A \cap\left(\left[-\frac{1}{n}, \frac{1}{n}\right] \times\left[-\frac{1}{m}, \frac{1}{m}\right]\right)\right)}{\frac{4}{n \cdot m}}>1-\frac{\epsilon}{4} .
$$

Put $\delta=\frac{1}{n_{0}}$. Let $h, k \in(0, \delta)$. Then $0<h<\delta=\frac{1}{n_{0}}$ and $0<k<\delta=\frac{1}{n_{0}}$, so there exist positive integers $n, m \geq n_{0}$ such that

$$
\frac{1}{n+1} \leq h<\frac{1}{n}
$$

and

Suppose that

$$
\frac{1}{m+1} \leq k<\frac{1}{m} \text {. }
$$

$$
f(h) \leq k \leq h
$$


Obviously,

$$
\frac{m_{2}(A \cap([-h, h] \times[-k, k]))}{4 h k}+\frac{m_{2}\left(A^{\prime} \cap([-h, h] \times[-k, k])\right)}{4 h k}=1 .
$$

We will prove that

$$
\frac{m_{2}\left(A^{\prime} \cap([-h, h] \times[-k, k])\right)}{4 h k}<\epsilon .
$$

Assume that $m>n$. Then

$$
\frac{1}{m} \leq \frac{1}{n+1}
$$

We have

$$
\begin{aligned}
& m_{2}\left(A^{\prime} \cap([-h, h] \times[-k, k])\right) \\
& \leq m_{2}\left(A^{\prime} \cap\left(\left[-\frac{1}{n}, \frac{1}{n}\right] \times\left[-\frac{1}{m}, \frac{1}{m}\right]\right)\right) \\
& \leq m_{2}\left(A^{\prime} \cap\left(\left[-\frac{1}{n+1}, \frac{1}{n+1}\right] \times\left[-\frac{1}{m}, \frac{1}{m}\right]\right)\right)+2\left(\frac{1}{n}-\frac{1}{n+1}\right) \cdot \frac{2}{m} \\
& =m_{2}\left(A^{\prime} \cap\left(\left[-\frac{1}{n+1}, \frac{1}{n+1}\right] \times\left[-\frac{1}{m}, \frac{1}{m}\right]\right)\right)+\frac{4}{n \cdot m \cdot(n+1)} .
\end{aligned}
$$

Hence

$$
\begin{aligned}
& \frac{m_{2}\left(A^{\prime} \cap([-h, h] \times[-k, k])\right)}{4 h k} \\
& \leq \frac{m_{2}\left(A^{\prime} \cap\left(\left[-\frac{1}{n+1}, \frac{1}{n+1}\right] \times\left[-\frac{1}{m}, \frac{1}{m}\right]\right)\right)}{4 \cdot \frac{1}{n+1} \cdot \frac{1}{m+1}}+\frac{4}{n \cdot m \cdot(n+1)} \cdot \frac{(n+1)(m+1)}{4} \\
& <\frac{m_{2}\left(A^{\prime} \cap\left(\left[-\frac{1}{n+1}, \frac{1}{n+1}\right] \times\left[-\frac{1}{m}, \frac{1}{m}\right]\right)\right)}{4 \cdot \frac{1}{n+1} \cdot \frac{1}{m}} \cdot \frac{m+1}{m}+\frac{2}{n} .
\end{aligned}
$$

From (4), (6), (5) and (7) we have

$$
f\left(\frac{1}{n+1}\right) \leq k<\frac{1}{m} \leq \frac{1}{n+1},
$$

so, from our assumption,

$$
\frac{m_{2}\left(A^{\prime} \cap\left(\left[-\frac{1}{n+1}, \frac{1}{n+1}\right] \times\left[-\frac{1}{m}, \frac{1}{m}\right]\right)\right.}{\frac{4}{(n+1) \cdot m}}<\frac{\epsilon}{4} .
$$




\section{ELŻBIETA WAGNER-BOJAKOWSKA — WŁADYSŁAW WILCZYŃSKI}

Clearly, $(m+1) / m \leq 2$ and $n_{0} \geq 4 / \epsilon$, so from (8) and (9),

$$
\frac{m_{2}\left(A^{\prime} \cap([-h, h] \times[-k, k])\right)}{4 h k}<\epsilon .
$$

If $f(k) \leq h \leq k$, the proof is analogous.

Let $\mathcal{S}$ denote the family of all measurable subsets of the plane.

Let $f \in C_{0}$ and $A \in \mathcal{S}$. $D_{f}(A)$ will denote the set of all points $(x, y) \in \mathbb{R}^{2}$ which are the density points of $A$ with respect to $f$.

Let $f, g \in C_{0}$ and $f(x) \leq g(x)$ for $x \in(0,1]$. It is clear that for arbitrary measurable subset $A$ of the plane, $D_{f}(A) \subset D_{g}(A)$.

Theorem 3. Let $f \in C_{0}$ and $c \in(0,1]$. For arbitrary measurable subset $A$ of the plane $D_{f}(A)=D_{c f}(A)$.

Pr o of. Let $A \in \mathcal{S}$. It is clear that $D_{c f}(A) \subset D_{f}(A)$, because if $c \in(0,1]$, then $c f(x) \leq f(x)$ for $x \in(0,1]$.

Now, we will prove that $D_{f}(A) \subset D_{c f}(A)$. Let $\epsilon>0$ and let $(x, y) \in D_{f}(A)$. The Lebesgue measure is invariant with respect to translation, so we may assume that $(x, y)=(0,0)$. There exists $\delta>0$ such that for each $h, k \in(0, \delta)$ if $f(h) \leq$ $k \leq h$ or $f(k) \leq h \leq k$, then

$$
\frac{m_{2}\left(A^{\prime} \cap([-h, h] \times[-k, k])\right)}{4 h k}<c \cdot \epsilon .
$$

Let $h, k \in(0, \delta)$ be such two numbers that

$$
c \cdot f(h) \leq k \leq h .
$$

If $f(h) \leq k \leq h$, then the inequality

$$
\frac{m_{2}\left(A^{\prime} \cap([-h, h] \times[-k, k])\right)}{4 h k}<\epsilon
$$

follows from (10). In the other case (i.e., $k<f(h)$ ), let us put $k_{1}=f(h)$. Then $k<k_{1}=f(h) \leq h<\delta$, so $k_{1}, h \in(0, \delta)$ and $f(h) \leq k_{1} \leq h$. Hence from (10) we obtain

$$
\frac{m_{2}\left(A^{\prime} \cap\left([-h, h] \times\left[-k_{1}, k_{1}\right]\right)\right)}{4 h k_{1}}<c \cdot \epsilon .
$$

From (11) we have $c \cdot k_{1} \leq k$, so

$$
\begin{aligned}
& \frac{m_{2}\left(A^{\prime} \cap([-h, h] \times[-k, k])\right)}{4 h k} \\
& \leq \frac{m_{2}\left(A^{\prime} \cap\left([-h, h] \times\left[-k_{1}, k_{1}\right]\right)\right)}{4 h k_{1}} \cdot \frac{k_{1}}{k} \\
& <c \cdot \epsilon \cdot \frac{k_{1}}{k} \leq \epsilon .
\end{aligned}
$$


If $c \cdot f(k) \leq h \leq k$, then the proof is analogous. Consequently, $(x, y) \in D_{c f}(A)$.

Definition 4. We will say that two functions $f, g \in C_{0}$ are equivalent if for arbitrary measurable set $A \subset \mathbb{R}^{2}$ we have $D_{f}(A)=D_{g}(A)$.

In this case, we will write $f \sim g$. From the previous theorem it follows that $f \sim c f$, for each $f \in C_{0}$ and $c \in(0,1]$. Particularly, if $g(x)=c \cdot x$, where $x \in(0,1]$ and $c \in(0,1]$, then for arbitrary measurable set $A \subset \mathbb{R}^{2}$, the set $D_{g}(A)$ of density points of $A$ with respect to $g$ coincides with the set of ordinary density points of $A$.

Let $A \in \mathcal{S} . \Phi_{0}(A)\left(\Phi_{s}(A)\right)$ will denote the set of ordinary (strong) density points of $A$. Obviously, for each function $f \in C_{0}$ and $A \in \mathcal{S}$

$$
\Phi_{s}(A) \subset D_{f}(A) \subset \Phi_{0}(A) .
$$

Let $A, B \in \mathcal{S}$. We write $A \sim B$ if and only if $m_{2}(A \triangle B)=0$ (where $A \triangle B=$ $(A \backslash B) \cup(B \backslash A))$.

Now, let $f \in C_{0}$ be a fixed function.

TheOREM 5. The mapping $D_{f}: S \rightarrow 2^{\mathbb{R}^{2}}$ has the following properties: for each $A, B \in \mathcal{S}$,

1) $A \sim D_{f}(A)$,

2) if $A \sim B$, then $D_{f}(A)=D_{f}(B)$,

3) $D_{f}(\emptyset)=\emptyset, D_{f}\left(\mathbb{R}^{2}\right)=\mathbb{R}^{2}$,

4) if $A \subset B$, then $D_{f}(A) \subset D_{f}(B)$,

5) $D_{f}(A \cap B)=D_{f}(A) \cap D_{f}(B)$.

Pr o of. 1) From (14), for arbitrary $A \in \mathcal{S}$ we obtain

$$
A \backslash D_{f}(A) \subset A \backslash \Phi_{s}(A),
$$

so, $m_{2}\left(A \backslash D_{f}(A)\right)=0$ (see [S, Theorem 10.2, p. 129]). Simultaneously,

$$
D_{f}(A) \backslash A \subset \Phi_{0}(A) \backslash A,
$$

so, $m_{2}\left(D_{f}(A) \backslash A\right)=0$ from Lebesgue density theorem for ordinary density. Consequently, $m_{2}\left(A \triangle D_{f}(A)\right)=0$, i.e., $A \sim D_{f}(A)$.

The conditions 2), 3) and 4) are obvious. 5) Clearly, $A \cap B \subset A$ and $A \cap B \subset B$, so from 4)

$$
D_{f}(A \cap B) \subset D_{f}(A) \cap D_{f}(B) .
$$

To prove the opposite inclusion, use the inequality

$$
\frac{m_{2}(A \cap I)}{m_{2}(I)}+\frac{m_{2}(B \cap I)}{m_{2}(I)}-1 \leq \frac{m_{2}(A \cap B \cap I)}{m_{2}(I)}
$$

which holds for arbitrary interval $I \subset \mathbb{R}^{2}$. 


\section{ELŻBIETA WAGNER-BOJAKOWSKA — WŁADYSŁAW WILCZYŃSKI}

In fact, $D_{f}: \mathcal{S} \rightarrow \mathcal{S}$, because from the first condition of the latter theorem, it follows that $D_{f}(A) \in \mathcal{S}$ for each $A \in \mathcal{S}$.

Let $\mathcal{T}_{0}$ and $\mathcal{T}_{s}$ denote the ordinary density topology and the strong density topology on the plane, respectively.

Put

$$
\mathcal{T}_{f}=\left\{A \in \mathcal{S}: A \subset D_{f}(A)\right\} .
$$

Obviously, $\mathcal{T}_{f} \subset \mathcal{S}$ and if $f \sim g$ then $\mathcal{T}_{f}=\mathcal{T}_{g}$.

From Theorem 5 it follows that the mapping $D_{f}$ is a lower density (see [LMZ]). Using Proposition 6.37 in [LMZ] and (14), we obtain the following result:

TheOREm 6. The family $\mathcal{T}_{f}$ is a topology on the plane, between ordinary and strong density topologies.

Observe that there exist functions $f \in C_{0}$ such that

$$
\mathcal{T}_{s} \varsubsetneqq \mathcal{T}_{f} \varsubsetneqq \mathcal{T}_{\text {, }}
$$

Put $f(x)=x^{2}$ for $x \in(0,1]$,

and

$$
\begin{aligned}
A=\{(x, y) & \in \mathbb{R}^{2}:\left[x>0 \text { and }\left(y>x^{3} \quad \text { or } y<-x^{3}\right)\right] \\
& \text { or } \left.\left[x<0 \text { and }\left(y>-x^{3} \quad \text { or } \quad y<x^{3}\right)\right]\right\} \cup\{(0,0)\}
\end{aligned}
$$

$$
B=\left\{(x, y) \in \mathbb{R}^{2}: y>x^{2} \quad \text { or } \quad y<-x^{2}\right\} \cup\{(0,0)\} .
$$

Then

$$
\lim _{h \rightarrow 0^{+}} \frac{m_{2}\left(A^{\prime} \cap\left([-h, h] \times\left[-h^{2}, h^{2}\right]\right)\right)}{4 h^{3}}=\lim _{h \rightarrow 0^{+}} \frac{h}{4}=0,
$$

so $(0,0) \in D_{f}(A)$ and $A \in \mathcal{T}_{f}$. Simultaneously,

$$
\lim _{h \rightarrow 0^{+}} \frac{m_{2}\left(A^{\prime} \cap\left([-h, h] \times\left[-h^{3}, h^{3}\right]\right)\right)}{4 h^{4}}=\frac{1}{4} .
$$

Consequently, $A \notin \mathcal{T}_{\text {s }}$.

Analogously,

$$
\lim _{h \rightarrow 0^{+}} \frac{m_{2}\left(B^{\prime} \cap([-h, h] \times[-h, h])\right)}{4 h^{2}}=\lim _{h \rightarrow 0^{+}} \frac{h}{3}=0,
$$

so $(0,0) \in \Phi_{0}(B)$ and $B \in \mathcal{T}_{0}$. On the other hand,

$$
\lim _{h \rightarrow 0^{+}} \frac{m_{2}\left(B^{\prime} \cap[-h, h] \times\left[-h^{2}, h^{2}\right]\right)}{4 h^{3}}=\frac{1}{3},
$$

so $(0,0) \notin D_{f}(B)$ and $B \notin \mathcal{T}_{f}$.

Essentially in the same way as in $[\mathrm{O}], \mathrm{Os}$, [Sch], [T] and [W], one can obtain the following results (compare also [LMZ, Theorem 6.39]). 
TheOREM 7. If $A$ is a measurable subset of the plane then

(a) $\operatorname{Int}_{\mathcal{T}_{f}}(A)=A \cap D_{f}(A)$,

(b) $\bar{A}^{\mathcal{T}_{f}}=A \cup\left[\mathbb{R}^{2} \backslash D_{f}\left(\mathbb{R}^{2} \backslash A\right)\right]$,

(c) $A^{d_{\mathcal{T}_{f}}}=\mathbb{R}^{2} \backslash D_{f}\left(\mathbb{R}^{2} \backslash A\right)$.

For arbitrary set $A \subset \mathbb{R}^{2}$,

$$
\operatorname{Int}_{\mathcal{T}_{f}}(A)=A \cap D_{f}(B),
$$

where $B$ is a measurable kernel of $A$.

Remark 8. If $m_{2}(A)=0$, then $A$ is closed in the topology $\mathcal{T}_{f}$.

Remark 9. If $A \in \mathcal{S}$ then $A \sim \operatorname{Int}_{\mathcal{T}_{f}} A$ and $A \sim \bar{A}^{\mathcal{T}_{f}}$.

TheOrem 10. The following conditions are equivalent:

(a) $m_{2}(A)=0$,

(b) $A$ is a nowhere dense set in the topology $\mathcal{T}_{f}$,

(c) $A$ is a set of the first category in the topology $\mathcal{T}_{f}$,

(d) $A^{d_{\mathcal{T}_{f}}}=\emptyset$.

TheOREM 11. The following conditions are equivalent:

(a) $A$ is measurable on the plane,

(b) $A$ is a Borel set in the topology $\mathcal{T}_{f}$,

(c) A has the Baire property in the topology $\mathcal{T}_{f}$.

ThEOREM 12. The set $A \subset \mathbb{R}^{2}$ is compact in the topology $\mathcal{T}_{f}$ if and only if A is finite.

P r o of. If $A$ is infinite, then there exists a countable set $A_{1} \subset A$ which is not compact in the natural topology. Hence, $A_{1}$ is not compact in the topology $\mathcal{T}_{f}$. Consequently, $A$ is not compact in the topology $\mathcal{T}_{f}$, because $A_{1}$ is closed in $\mathcal{T}_{f}$. The inverse implication is obvious.

Theorem 13. The space $\left(\mathbb{R}^{2}, \mathcal{T}_{f}\right)$ is neither first countable, nor second countable, nor Lindelöf, nor separable.

TheOREM 14. $\left(\mathbb{R}^{2}, \mathcal{T}_{f}\right)$ is a Baire space.

P r o of. It is obvious because each non-empty set open in the topology $\mathcal{T}_{f}$ has positive measure.

TheOREM 15. $\left(\mathbb{R}^{2}, \mathcal{T}_{f}\right)$ is a regular space. 


\section{ELŻBIETA WAGNER-BOJAKOWSKA — WŁADYSŁAW WILCZYŃSKI}

The proof using Theorem 2 is analogous to the proof of Theorem 22.9 in [O]. From (17) we obtain

$$
\mathcal{T}_{s} \subset \bigcap_{f \in C_{0}} \mathcal{T}_{f} \subset \bigcup_{f \in C_{0}} \mathcal{T}_{f} \subset \mathcal{T}_{0} .
$$

On the other hand, $\mathcal{T}_{0} \subset \bigcup_{f \in C_{0}} \mathcal{T}_{f}$, since $\mathcal{T}_{0}$ is a $\mathcal{T}_{f}$ topology for a function $f(x)=x, x \in(0,1]$, and $f \in C_{0}$. Consequently,

$$
\bigcup_{f \in C_{0}} \mathcal{T}_{f}=\mathcal{T}_{0} .
$$

TheOREM 16. $\bigcap_{f \in C_{0}} \mathcal{T}_{f}=\mathcal{T}_{s}$.

Proof. It is sufficient to prove that

$$
\bigcap_{f \in C_{0}} \mathcal{T}_{f} \subset \mathcal{T}_{s} .
$$

Let $A \in \bigcap_{f \in C_{0}} \mathcal{T}_{f}$ and let $\left(x_{0}, y_{0}\right) \in A$. We will prove that for arbitrary sequence $\left\{P_{n}\right\}_{n \in \mathbb{N}}$ of rectangles centered at $\left(x_{0}, y_{0}\right)$ such that $\delta\left(P_{n}\right) \underset{n \rightarrow \infty}{\rightarrow} \longrightarrow 0$, we have

$$
\lim _{n \rightarrow \infty} \frac{m_{2}\left(A \cap P_{n}\right)}{m_{2}\left(P_{n}\right)}=1 .
$$

Let $\left\{P_{n}\right\}_{n \in \mathbb{N}}$ be an arbitrary sequence of such intervals. We can assume that $\left(x_{0}, y_{0}\right)=(0,0)$ and all intervals $P_{n}, n \in \mathbb{N}$, are contained in $(-1,1) \times(-1,1)$. We divide the sequence $\left\{P_{n}\right\}_{n \in \mathbb{N}}$ into two subsequences $\left\{P_{n}^{1}\right\}_{n \in \mathbb{N}}$ and $\left\{P_{n}^{2}\right\}_{n \in \mathbb{N}}$ in the following way: in the first sequence there are all intervals $P_{n}=\left[-h_{n}, h_{n}\right] \times$ $\left[-k_{n}, k_{n}\right]$, for which $h_{n}>k_{n}$, and in the second sequence there are all the others. We will prove that

$$
\lim _{n \rightarrow \infty} \frac{m_{2}\left(A \cap P_{n}^{1}\right)}{m_{2}\left(P_{n}^{1}\right)}=1 .
$$

Let $\left\{P_{n}^{3}\right\}_{n \in \mathbb{N}}$ be an arbitrary subsequence of $\left\{P_{n}^{1}\right\}_{n \in \mathbb{N}}$. Put $P_{n}^{3}=\left[-x_{n}, x_{n}\right] \times$ $\left[-y_{n}, y_{n}\right], n \in \mathbb{N}$. From the sequence $\left\{x_{n}\right\}_{n \in \mathbb{N}}$ we can choose the subsequence $\left\{x_{n_{k}}\right\}_{k \in \mathbb{N}}$ tending decreasingly to zero, and then, from the sequence $\left\{y_{n_{k}}\right\}_{k \in \mathbb{N}}$ we choose the subsequence $\left\{y_{n_{k_{l}}}\right\}_{l \in \mathbb{N}}$ tending decreasingly to zero. Put

$$
P_{l}^{4}=\left[-x_{n_{k_{l}}}, x_{n_{k_{l}}}\right] \times\left[-y_{n_{k_{l}}}, y_{n_{k_{l}}}\right]
$$

for $l \in \mathbb{N}$. Obviously, $\left\{P_{n}^{4}\right\}_{n \in \mathbb{N}}$ is a subsequence of $\left\{P_{n}^{3}\right\}_{n \in \mathbb{N}}$. Let $f$ be a function defined as follows: $f(1)=1, f\left(x_{n_{k_{l}}}\right)=y_{n_{k_{l}}}$ for $l \in \mathbb{N}$ and $f$ is linear on the intervals $\left[x_{1}, 1\right],\left[x_{n_{k_{l+1}}}, x_{n_{k_{l}}}\right], l \in \mathbb{N}$. Obviously, $f \in C_{0}$, so, from our assumption,

$$
\lim _{n \rightarrow \infty} \frac{m_{2}\left(A \cap P_{n}^{4}\right)}{m_{2}\left(P_{n}^{4}\right)}=1 .
$$




\section{DENSITY TOPOLOGIES ON THE PLANE BETWEEN ORDINARY AND STRONG}

From Urysohn condition we obtain (19). We can analogously deal with the sequence $\left\{P_{n}^{2}\right\}_{n \in \mathbb{N}}$ obtaining the function $g \in C_{0}$. Hence,

$$
\lim _{n \rightarrow \infty} \frac{m_{2}\left(A \cap P_{n}^{2}\right)}{m_{2}\left(P_{n}^{2}\right)}=1 .
$$

Consequently, we have (18), so $(0,0) \in \Phi_{s}(A)$ and $A \in \mathcal{T}_{s}$.

Theorem 17. Let $f, g \in C_{0}$ and $f(x) \leq g(x)$ for $x \in(0,1]$. If there exists a number $c \in(0,1)$ such that

then $\mathcal{T}_{f}=\mathcal{T}_{g}$.

$$
\liminf _{x \rightarrow 0^{+}} \frac{f(x)}{g(x)}=c,
$$

Proof. Let $f, g \in C_{0}$ and $f(x) \leq g(x)$ for $x \in(0,1]$. From our assumption there exists $\delta_{1}>0$ such that

$$
\frac{f(x)}{g(x)}>\frac{c}{2}
$$

for $x \in\left(0, \delta_{1}\right)$. We will prove that $D_{\frac{c}{2} g}(A) \subset D_{f}(A)$ for arbitrary $A \in \mathcal{S}$.

Let $A \in \mathcal{S}$ and let $(0,0) \in D_{\frac{c}{2} g}(A)$. There exists $\delta_{2}>0$ such that for each $h, k \in\left(0, \delta_{2}\right)$ if $\frac{c}{2} g(h) \leq k \leq h$ or $\frac{c}{2} g(k) \leq h \leq k$, then

$$
\frac{m_{2}\left(A^{\prime} \cap[-h, h] \times[-k, k]\right)}{4 h k}<\epsilon .
$$

Put $\delta_{0}=\min \left(\delta_{1}, \delta_{2}\right)$. Let $h, k \in\left(0, \delta_{0}\right)$ be two numbers such that $f(h) \leq k \leq h$. Then $h \in\left(0, \delta_{1}\right)$, so

$$
\frac{c}{2} g(h) \leq f(h) \leq k \leq h
$$

and $h, k \in\left(0, \delta_{2}\right)$. Consequently, the condition (20) is fulfilled. Analogously, if $f(k) \leq h \leq k$. Hence, $(0,0) \in D_{f}(A)$.

Obviously, $D_{f}(A) \subset D_{g}(A)$ for $A \in \mathcal{S}$. From Theorem 3 we have $D_{\frac{c}{2} g}(A)=$ $D_{g}(A)$ for $A \in \mathcal{S}$. Consequently, $D_{f}(A)=D_{g}(A)$ for each $A \in \mathcal{S}$. Hence, $f \sim g$ and $\mathcal{T}_{f}=\mathcal{T}_{g}$.

Corollary 18. If $f \in C_{0}$ and $\liminf _{x \rightarrow 0^{+}} \frac{f(x)}{x}>0$, then $\mathcal{T}_{f}=\mathcal{T}_{0}$.

Now, let $A$ be a subset of a real line and let $x \in \mathbb{R}$. Let $p^{+}(A, x)$ denote the right-hand porosity of $A$ at $x$, i.e.,

$$
p^{+}(A, x)=\limsup _{h \rightarrow 0^{+}} \frac{\lambda(A,(x, x+h))}{h},
$$

where $\lambda(A, I)=\sup \{m(J): J \subset I \backslash A\}$.

Let $f, g \in C_{0}$ and $c \in(0,1)$. Put

$$
M_{c}=\{x \in(0,1]: g(x) \geq c \cdot f(x)\} .
$$




\section{ELŻBIETA WAGNER-BOJAKOWSKA — WŁADYSŁAW WILCZYŃSKI}

Definition 19. We say that the pair $(f, g)$ fulfills the condition $(*)$ if for each decreasing sequence $\left\{c_{n}\right\}_{n \in \mathbb{N}}$ convergent to zero

$$
\lim _{n \rightarrow \infty} p^{+}\left(M_{c_{n}}, 0\right)=0 .
$$

Observe that if $c_{1}, c_{2} \in(0,1], c_{1}<c_{2}$ then $M_{c_{1}} \supset M_{c_{2}}$. Consequently, the condition $(*)$ is equivalent to the following

$$
\text { there exists }\left\{c_{n}\right\} \searrow 0 \text { such that } \lim _{n \rightarrow \infty} p^{+}\left(M_{c_{n}}, 0\right)=0 \text {. }
$$

The implication $(*) \Longrightarrow(* *)$ is obvious. Now, assume $(* *)$ and let $\left\{d_{n}\right\}_{n \in \mathbb{N}}$ be an arbitrary decreasing sequence convergent to zero. Then there exists $n_{0} \in \mathbb{N}$ such that for each $n \geq n_{0}$ there exists $k_{n} \in \mathbb{N}$ such that $d_{n} \leq c_{k_{n}}$. Hence $M_{d_{n}} \supset M_{c_{k_{n}}}$, so

$$
p^{+}\left(M_{d_{n}}, 0\right) \leq p^{+}\left(M_{c_{k_{n}}}, 0\right) .
$$

Consequently, $\lim _{n \rightarrow \infty} p^{+}\left(M_{d_{n}}, 0\right)=0$.

Let $f \in C_{0}$. Due to the simplification, we use the intervals of the form $[0, x] \times$ $[0, y]$, where $y \leq x, x>0, y>0$.

Let $f, g \in C_{0}$ and $g(x) \leq f(x)$ for $x \in(0,1]$. If $A \subset[0, \infty) \times[0, \infty)$ is a measurable set fulfilling the condition

$$
\lim _{\substack{(x, y) \rightarrow 0 \\ g(x) \leq y \leq x}} \frac{m_{2}(A \cap([0, x] \times[0, y]))}{x \cdot y}=0
$$

then, obviously,

$$
\lim _{\substack{(x, y) \rightarrow 0 \\ f(x) \leq y \leq x}} \frac{m_{2}(A \cap([0, x] \times[0, y]))}{x \cdot y}=0 .
$$

The next theorem gives some sufficient condition for which the implication $(22) \Longrightarrow$ (21) holds.

TheORem 20. Let $f, g \in C_{0}, g(x) \leq f(x)$ for $x \in(0,1]$. If the pair $(f, g)$ fulfills the condition $(*)$, then the conditions (21) and (22) are equivalent.

P r o o f. Let $A \subset[0, \infty) \times[0, \infty)$ be a measurable set. Let $f, g \in C_{0}, g(x) \leq f(x)$ for $x \in(0,1]$ and the pair $(f, g)$ fulfills $(*)$. Suppose that (22) is fulfilled. We will prove that (21) also holds.

Let $\epsilon>0$ and let $\left\{c_{n}\right\}_{n \in \mathbb{N}}$ be an arbitrary decreasing sequence tending to zero. From $(*)$ there exists $n_{0} \in \mathbb{N}$ such that

$$
p^{+}\left(M_{c_{n_{0}}}, 0\right)<\frac{\epsilon}{3} .
$$

Hence, there exists $\delta_{1}>0$ such that if $h \in\left(0, \delta_{1}\right)$ and $(a, b) \subset(0, h) \cap M_{c_{n_{0}}}^{\prime}$, then $(b-a) / b<\epsilon / 2$. 


\section{DENSITY TOPOLOGIES ON THE PLANE BETWEEN ORDINARY AND STRONG}

We may assume that $\delta_{1} \in M_{c_{n_{0}}}$. From (22) it follows that there exists $\delta_{2}>0$ such that if $\rho((x, y),(0,0))<\delta_{2}$ and $f(x) \leq y \leq x$. Then

$$
\frac{m_{2}(A \cap([0, x] \times[0, y]))}{x \cdot y}<\frac{\epsilon c_{n_{0}}}{2} .
$$

Since $f(x) \leq x$, so, for $x \in\left(0, \delta_{2} / \sqrt{2}\right)$, we have $\rho((x, f(x)),(0,0))<\delta_{2}$. Consequently,

$$
\frac{m_{2}(A \cap([0, x] \times[0, f(x)]))}{x \cdot f(x)}<\frac{\epsilon c_{n_{0}}}{2} .
$$

We may assume that $\delta_{2} / \sqrt{2} \in M_{c_{n_{0}}}$.

Put $\delta=\min \left(\delta_{1}, \delta_{2} / \sqrt{2}\right)$. We will prove that if $x \in(0, \delta)$ and $g(x) \leq y \leq x$, then

$$
\frac{m_{2}(A \cap([0, x] \times[0, y]))}{x \cdot y}<\epsilon
$$

i.e., the condition (21) holds. There are two possibilities:

$1^{o} x \in(0, \delta) \cap M_{c_{n_{0}}}$. Then, if $f(x) \leq y \leq x$, the inequality (25) follows from (23) $\left(c_{n_{0}}<1\right)$. Suppose that $g(x) \leq y<f(x)$. We have $x \in M_{c_{n_{0}}}$, so $g(x) \geq c_{n_{0}} \cdot f(x)$ and $c_{n_{0}} f(x) \leq y<f(x)$. Consequently, from (24) we obtain

$$
\frac{m_{2}(A \cap([0, x] \times[0, y]))}{x \cdot y} \leq \frac{m_{2}(A \cap([0, x] \times[0, f(x)]))}{x \cdot c_{n_{0}} f(x)}<\frac{\epsilon}{2} .
$$

$2^{o} \quad x \in(0, \delta) \cap M_{c_{n_{0}}}^{\prime}$. Obviously, $M_{c_{n_{0}}}^{\prime}$ is an open set, so there exists a component interval $(a, b)$ of $M_{c_{n_{0}}}^{\prime}$ such that $x \in(a, b)$. Then $(a, x) \subset\left(0, \delta_{1}\right) \cap M_{c_{n_{0}}}^{\prime}$ and, consequently, $(x-a) / x<\epsilon / 2$. Clearly, $a \in M_{c_{n_{0}}}$, so $g(a) \geq c_{n_{0}} \cdot f(a)$. Simultaneously for $t \in(a, x)$ we have $g(t)<c_{n_{0}} \cdot f(t)$, so, from the continuity of $f$ and $g$, we obtain $g(a)=c_{n_{0}} \cdot f(a)$.

Now, let $g(x) \leq y \leq x$. Obviously, $g(a) \leq y$. If $y \leq a$ then $g(a) \leq y \leq a$ and $a \in(0, \delta) \cap M_{c_{n_{0}}}$, so, from $1^{0}$, we obtain

$$
\frac{m_{2}(A \cap([0, a] \times[0, y]))}{a \cdot y}<\frac{\epsilon}{2} .
$$

Consequently, using (27), we have

$$
\begin{aligned}
\frac{m_{2}(A \cap([0, x] \times[0, y]))}{x \cdot y} & \leq \frac{m_{2}(A \cap([0, a] \times[0, y]))}{x \cdot y}+\frac{(x-a) y}{x \cdot y} \\
& \leq \frac{m_{2}(A \cap([0, a] \times[0, y]))}{a \cdot y}+\frac{x-a}{x}<\epsilon,
\end{aligned}
$$

because $(a, x) \subset\left(0, \delta_{1}\right) \cap M_{c_{n_{0}}}^{\prime}$. 


\section{ELŻBIETA WAGNER-BOJAKOWSKA — WŁADYSŁAW WILCZYŃSKI}

If $a<y$, then $a<y \leq x<\delta \leq \delta_{2} / \sqrt{2}$, so $\rho((y, y),(0,0))<\delta_{2}, f(y) \leq y$ and from (23) we obtain

$$
\begin{aligned}
\frac{m_{2}(A \cap([0, x] \times[0, y]))}{x \cdot y} & \leq \frac{m_{2}(A \cap([0, y] \times[0, y]))}{x \cdot y}+\frac{(x-y) y}{x \cdot y} \\
& \leq \frac{m_{2}(A \cap([0, y] \times[0, y]))}{y^{2}}+\frac{x-a}{x}<\epsilon,
\end{aligned}
$$

because $(y, x) \subset(a, x) \subset\left(0, \delta_{1}\right) \cap M_{c_{n_{0}}}^{\prime}$.

Finally, from (26), (28) and (29) we obtain (21).

Corollary 21. Let $f, g \in C_{0}, g(x) \leq f(x)$ for $x \in(0,1]$. If the pair $(f, g)$ fulfills the condition $(*)$ then for arbitrary measurable set $A \subset \mathbb{R}^{2}$, we have $D_{f}(A)=D_{g}(A)$, so $f \sim g$ and $\mathcal{T}_{f}=\mathcal{T}_{g}$.

Remark 22. There exist functions $f, g \in C_{0}$ such that $\mathcal{T}_{f} \backslash \mathcal{T}_{g} \neq \emptyset$ and $\mathcal{T}_{g} \backslash \mathcal{T}_{f} \neq \emptyset$.

Take $q \in(0,1)$. Put

and

$$
f_{0}(x)=\left\{\begin{array}{lll}
\frac{1}{1 !} & \text { for } x \in\left[q^{1}, q^{0}\right] \\
\frac{1}{(2 n+1) !} & \text { for } x \in\left[q^{4 n+1}, q^{4 n-2}\right], & n \geq 1, \\
\text { linear and continuous on }\left[q^{4 n-2}, q^{4 n-3}\right], & n \geq 1
\end{array}\right.
$$

$$
g_{0}(x)= \begin{cases}\frac{1}{(2 n+2) !} \quad \text { for } x \in\left[q^{4 n+3}, q^{4 n}\right], & n \geq 0 \\ \text { linear and continuous on }\left[q^{4 n}, q^{4 n-1}\right], & n \geq 1\end{cases}
$$

It is not difficult to see that there exists $c>0$ such that $c \cdot f_{0}(x) \leq x$ and $c \cdot g_{0}(x) \leq x$ for $x \in(0,1]$. Put $f=c \cdot f_{0}$ and $g=c \cdot g_{0}$.

Observe that

and

$$
\frac{f(x)}{g(x)}=2 n+2 \text { for } x \in\left[q^{4 n+1}, q^{4 n}\right], \quad n \geq 0
$$

$$
\frac{g(x)}{f(x)}=2 n+3 \text { for } x \in\left[q^{4 n+3}, q^{4 n+2}\right], \quad n \geq 0 .
$$

Let

$$
A=\bigcup_{n=1}^{\infty}\left[q^{4 n-1}, q^{4 n-2}\right] \times\left[0, f\left(q^{4 n-1}\right)\right] \text { and } B=\bigcup_{n=0}^{\infty}\left[q^{4 n+1}, q^{4 n}\right] \times\left[0, g\left(q^{4 n+1}\right)\right] \text {. }
$$

Observe that for $h=q^{4 n-2}, k=f\left(q^{4 n-2}\right), n \geq 1$ we have

$$
\frac{m_{2}(A \cap([-h, h] \times[-k, k]))}{4 h k} \geq \frac{1}{4} \cdot \frac{q^{4 n-2}-q^{4 n-1}}{q^{4 n-2}}=\frac{1}{4}(1-q)>0,
$$

so $(0,0)$ is not a density point of $\mathbb{R}^{2} \backslash A$ with respect to $f$ and $\mathbb{R}^{2} \backslash A \notin \mathcal{T}_{f}$. Simultaneously, for $h \in\left[q^{4 n+3}, q^{4 n-1}\right]$ and $g(h) \leq k \leq h$ we have 


\section{DENSITY TOPOLOGIES ON THE PLANE BETWEEN ORDINARY AND STRONG}

$$
\frac{m_{2}(A \cap([-h, h] \times[-k, k]))}{4 h k} \leq \frac{1}{4} \cdot \frac{1}{2 n+3}
$$

because $A \cap([-h, h] \times[-k, k]) \subset[0, h] \times\left[0, f\left(q^{4 n+3}\right)\right]$ and $\frac{g(h)}{f\left(q^{4 n+3}\right)} \geq 2 n+3$ for $h \in\left[q^{4 n+3}, q^{4 n-1}\right]$. Hence, $(0,0)$ is a density point of $\mathbb{R}^{2} \backslash A$ with respect to $g$. Observe also that $\mathbb{R}^{2} \backslash(A \cup\{(0,0)\})$ is open in the natural topology on the plane, so $\mathbb{R}^{2} \backslash A \subset D_{g}\left(\mathbb{R}^{2} \backslash A\right)$ and $\mathbb{R}^{2} \backslash A \in \mathcal{T}_{g}$. Similarly, one can prove that $\mathbb{R}^{2} \backslash B \in \mathcal{T}_{f} \backslash \mathcal{T}_{g}$.

\section{REFERENCES}

[LMZ] LUKEŠ, J.-MALÝ, J.-ZAJÍČEK, L.: Fine Topology Methods in Real Analysis and Potential Theory, Lecture Notes in Math., Vol. 1189, Springer-Verlag, Berlin, 1986.

[O] OXTOBY, J. C.: Measure and Category. A Survey of the Analogies Between Topological and Measure Spaces (2nd ed.). Grad. Texts in Math., Vol. 2, Springer-Verlag, New York, 1980.

[Os] OSTASZEWSKI, K.: Continuity in the density topology, Real Anal. Exchange 7 (1982), 259-270.

[S] SAKS, S.: Theory of the Integral. Hafner Publ. Comp., New York, 1938.

[Sch] SCHEINBERG, S.: Topologies which generate a complete measure algebra, Adv. Math. 7 (1971), 231-239.

[T] TALL, F. D.: The density topology, Pacific J. Math. 62 (1976), 275-284.

[W] WILCZYŃSKI, W.: Density topologies, in: Handbook of Measure Theory (E. Pap, ed.), North-Holland, Amsterdam, 2002, pp. 675-702.

Elżbieta Wagner-Bojakowska

Faculty of Mathematics and

Computer Science

Banacha 22

PL-90-238 Eódź

POLAND

The College of Computer Science

Chair of Mathematics and Physics

Rzgowska $17 \mathrm{~A}$

PL-93-008 Eódź

POLAND

E-mail:wagner@uni.lodz.pl

Wtadystaw Wilczyński

Faculty of Mathematics and

Computer Science

Banacha 22

PL-90-238 Eódź

POLAND

E-mail: wwil@uni.lodz.pl 